\title{
Multiobjective Topology Optimization of Spatial-Structure Joints
}

\author{
Nanhai Zhu $\mathbb{( D D}^{1,2}$ and Jinlei Liu $\mathbb{D}^{1,2}$ \\ ${ }^{1}$ School of Civil and Surveying Engineering, Jiangxi University of Science and Technology, Ganzhou 341000, China \\ ${ }^{2}$ Jiangxi Provincial Key Laboratory of Environmental Geotechnical Engineering and Disaster Control, Ganzhou 341000, China
}

Correspondence should be addressed to Nanhai Zhu; zhunanhai@jxust.edu.cn

Received 6 January 2021; Revised 16 March 2021; Accepted 29 March 2021; Published 12 April 2021

Academic Editor: Xuemei Liu

Copyright (C) 2021 Nanhai Zhu and Jinlei Liu. This is an open access article distributed under the Creative Commons Attribution License, which permits unrestricted use, distribution, and reproduction in any medium, provided the original work is properly cited.

\begin{abstract}
To realize the static and dynamic multiobjective topology optimization of joints in spatial structures, structural topology optimization is carried out to maximize the stiffness under static multiload conditions and maximize the first third-order dynamic natural frequencies. According to the single-objective optimization results, the objective function of the multiobjective topology optimization of joints is established by using the compromise programming method, and the weight coefficient of each static load condition is determined by using the analytic hierarchy process. Subsequently, under the constraint of the volume fraction, the multiobjective topology optimization of joints is realized by minimizing the multiobjective function. Finally, the optimized structure is smoothed to obtain a smoother joint, and its mechanical properties are compared with those of the hollow ball joint. The results indicate that the multiobjective topology optimization that considers the static stiffness and dynamic frequency can effectively improve the mechanical properties of the structure. Through the research on multiobjective topology optimization, a new type of spatial joint with reasonable stress, a novel form, and aesthetic shape can be obtained, which mitigates the shortcomings of single-objective topology optimization. In comparison to hollow spherical joints with the same weight, topologyoptimized joints have a superior ability to resist deformation and improve low-order frequency, which verifies the feasibility of applying multiobjective topology optimization to the lightweight design of joints.
\end{abstract}

\section{Introduction}

Joints are the key components that interconnect structural elements, such as large-span spatial trusses, grids, and reticulated shells [1-5]. The amount of steel used in joints that are connected to form a structural system often comprises $15 \%$ to $45 \%$ of the total structural steel consumption [6]. Therefore, topology optimization is an effective way to reduce the weight of the structure and improve its mechanical properties. In comparison to other optimization methods, this method has greater benefits and broader applicability [7-9].

Researchers have proposed different theoretical methods for the topology optimization of structural joints, which mainly focus on the single-objective optimization design of structures. Chen et al.[10] studied the topology optimization of joints under single- and multiple-load conditions and obtained models for the spatial-structure joints in additive manufacturing by averaging different load conditions. Further, $\mathrm{Li}$ et al.[11] analyzed the topology optimization of joints to maximize stiffness and minimize mass, respectively. Ye et al.[12] performed topology optimization of single-layer spatial grid structure joints under complex load conditions and solved the singularity of the stress matrix by applying the velocity field, which verified the advantages of the optimization design method for rigid joints by combining topology optimization with structural design. Wang et al.[13] studied the topology optimization of treelike joints with the goal of minimizing compliance and manufactured them through additive manufacturing. Seifi et al. [14] studied the topology optimization of structural joints of Sunshine Valley of World Expo under axial loading. Bai et al. [15] presents an explicit three-dimensional topology optimization method to obtain the hollow structures using moving morphable components (MMCs), which is achieved by combining two topology description functions, namely, internal and external topology 
description functions. Zuo et al. [16] exploit a semirigid beam element (SRBE) that consists of a beam element with two semirigid connections at the ends to simulate the flexibility of joint and verified the effectiveness and practicability of multithreaded parallel especially for frequency optimization. These studies have promoted the development of the topology optimization of spatial-structure joints and informed the application of topology optimization to structural joints. However, the studies only consider a single goal and usually adopt an average distribution method for multiple-load conditions. For spatial joints, the actual load conditions include axial load, shear load, and bending moment load with different weightings. Therefore, it is necessary to use multiobjective topology optimization to obtain joints suitable for engineering practice.

To mitigate the aforementioned limitations, this paper proposes a multiobjective topology optimization method for spatial-structure joints based on the compromise programming method and combines the analytic hierarchy process (AHP) to determine the weights of the three load conditions axial load, shear load, and bending moment load. Next, the topology optimization of the spatial grid structure joint is carried out to maximize structural stiffness and low-order frequencies, and the initial optimization result is smoothed to obtain a new joint type that meets multiple goals and simultaneously has reasonable stress transmission.

\section{Variable Density Method}

Structural topology optimization is a mathematical method to rationally distribute materials in a design area. These methods include the homogenization method [17], variable density method [18], independent, continuous, mapping (ICM) method [19], evolutionary structural optimization method (ESO) [20], moving morphable components (MMCs) [15], and bidirectional evolutionary structural optimization method (BESO) [21], among which the variable density method is widely used owing to its ease of calculation, fast convergence, and high solution accuracy. The variable density method artificially introduces a material whose density varies from 0 to 1 . The existence of the material is described by the numerical value. In this way, the topology optimization problem is transformed into a problem regarding the optimal allocation problem of materials. There are two interpolation models commonly used in the variable density method: solid isotropic material with penalization (SIMP) [22] and rational approximation of material properties (RAMP) [23]. Engineering application experience shows that the SIMP interpolation model is relatively simple and more commonly used [10]. Since the material density in the variable density method is artificially assumed, it is necessary to treat materials with intermediate densities. In the SIMP numerical model, the penalty coefficient $P$ is introduced to mitigate the checkerboard phenomenon after optimization, which greatly reduces the materials with intermediate densities in the structure. The SIMP interpolation model can be expressed as follows:

$$
E(\boldsymbol{\rho})=E_{\min }+\boldsymbol{\rho}^{P}\left(E_{0}-E_{\min }\right),
$$

where $\rho$ is the material density, $E(\rho)$ is the elastic modulus, $P$ is the penalty factor, $E_{0}$ and $E_{\min }$ are the elastic moduli of materials with unit densities approximately 1 and 0 , respectively, and $E_{\min }=E_{0} / 1000$ is usually assumed.

\section{Multiobjective Topology Optimization}

3.1. Maximum Stiffness Numerical Model. The topology optimization of static structures is a way to reasonably distribute materials in the design area to ensure maximum stiffness. In practice, joints are generally in complex stress states that include axial, shear, and bending moment stresses. Therefore, the static topology optimization problem aims to maximize the stiffness of the structure under multiple-load conditions, also known as the multistiffness topology optimization problem [24]. For static multiload condition topology optimization problems, each load corresponds to an optimal solution aimed at maximizing stiffness. The traditional solution converts multiload problems into single-load problems by using the linear weighting method, which is relatively simple. However, for nonconvex functions, this method cannot guarantee all Pareto optimal solutions. In this paper, the compromise programming method [25] is used to transform the static multiload condition objective function into a single-objective function.

By combining topology optimization and the compromise programming method, the minimum flexibility is used instead of the maximum stiffness, and the volume fraction is taken as a constraint. The static multiload condition mathematical function is as follows:

$$
\left\{\begin{array}{l}
\text { find } \boldsymbol{\rho}=\left\{\boldsymbol{\rho}_{1}, \boldsymbol{\rho}_{2}, \ldots, \boldsymbol{\rho}_{h}\right\}^{T}, \\
\min C(\boldsymbol{\rho})=\left[\sum_{k=1}^{m} \omega_{k}^{P}\left(\frac{C_{k}(\boldsymbol{\rho})-C_{k}^{\min }}{C_{k}^{\max }-C_{k}^{\min }}\right)^{P}\right]^{\frac{1}{P}}, \\
\frac{\text { s.t. } V(\boldsymbol{\rho})}{V_{0} \leq f}, \\
\mathbf{K}(\boldsymbol{\rho}) \mathbf{U}_{k}=\mathbf{F}_{k}, k=1,2, \ldots, m,
\end{array}\right.
$$

where $n$ is the total number of design area units, $m$ is the number of static loads, $C_{k}^{\min }$ and $C_{k}^{\max }$ are the minimum and maximum values of compliance when the $k$-th load condition is optimized, respectively, $C_{k}(\rho)$ is the compliance value of the $k$-th load condition, $\omega_{k}$ is the weight value of the $k$-th load condition, $V(\rho)$ is the optimized volume of the design area, $V_{0}$ is the initial volume of the design area, $f$ is the design volume fraction constraint value, $\mathbf{K}(\rho)$ is the element stiffness matrix, $\mathbf{U}_{k}$ is the element displacement vector, and $\mathbf{F}_{k}$ is the load vector.

3.2. Maximize Frequency Numerical Function. The purpose of structural dynamic natural frequency topology 
optimization is to increase the low-order natural frequencies of the structure. The reciprocal of the weighted frequencies is often used to consider the topology optimization of the loworder natural frequencies of the structure, the weighted sum of the reciprocals of the frequencies of each order is as follows:

$$
\begin{aligned}
\delta & =\sum \frac{\boldsymbol{\sigma}_{r}}{\lambda_{r}}, \\
\left(\mathbf{K}-\lambda_{r} \mathbf{M}\right) \mathbf{u}_{r} & =0,
\end{aligned}
$$

where $\delta$ is the reciprocal weighted sum of the frequencies of each order, $\sigma_{r}$ is the weight of the $r$-th frequency, $\lambda_{r}$ is the characteristic value of order $r, \mathbf{M}$ is the mass matrix, and $\mathbf{u}_{r}$ is the $r$-th mode displacement vector.

It can be seen from equation (3) that the low-order natural frequencies of the structure have a great influence on the optimization results. Owing to the frequency of each order not being positively correlated in the optimization process, it is possible that when the frequency of one order reaches the maximum, the frequency of other orders will decrease to a lower value, and even lead to the order exchange between different orders, causing a vibration problem of the objective function [26]. To solve this problem and increase the frequencies of each order of the structure, the average frequencies formula is used to define the objective function of natural frequency topology optimization [27]:

$$
\left\{\begin{array}{l}
\text { find } \boldsymbol{\rho}=\left\{\boldsymbol{\rho}_{1}, \boldsymbol{\rho}_{2}, \ldots, \boldsymbol{\rho}_{n}\right\}^{T}, \\
\max \Lambda(\boldsymbol{\rho})=\lambda_{0}+\frac{1}{\eta}\left(\sum_{r=1}^{s} \frac{\boldsymbol{\sigma}_{r}}{\lambda_{r}-\lambda_{0}}\right)^{-1}, \\
\frac{\text { s.t. } V(\boldsymbol{\rho})}{V_{0} \leq f}, \\
\left(\mathbf{K}(\boldsymbol{\rho})-\lambda_{r} \mathbf{M}(\boldsymbol{\rho})\right) \beta_{r}=0, r=1,2, \ldots, D_{e},
\end{array}\right.
$$

where $\Lambda(\rho)$ is the objective function of dynamic natural frequency optimization, $\lambda_{0}$ and $\eta$ are given parameters, $s$ is the order of structural frequency to be optimized, $\mathbf{M}(\rho)$ is the unit mass matrix, $\beta_{r}$ is the eigenvector corresponding to the $r$-th eigenvalue, $D_{e}$ is the total degree of freedom of the design area, and $\eta=1$ and $\lambda_{0}=0$.

3.3. Multiobjective Topology Optimization. Multiobjective topology optimization methods can be divided into two types. The first transforms the multiobjective problem into one or a series of single-objective problems, and the optimized result is regarded as the solution of the multiobjective optimization problem. The second is to directly seek noninferior solutions and choose the better solution as the optimal solution.

Both stiffness and frequency goals need to be considered when carrying out multiobjective topology optimization on a structure to ensure that the low-order natural frequency and structural stiffness are sufficiently high. The main issue with multiobjective optimization lies in how to unify the dimensions of multiple objective functions. In this study, the compromise programming method is used to first solve the single-objective problem and obtain the optimal value, which is called the ideal point. Then, the multiobjective solution is undertaken according to the single-objective optimized result, and the weighted objective function is constructed to find the minimum distance from the ideal point. Taking the minimization of the weighted objective function as the goal, and the volume fraction as the constraint, according to equations (2) and (4), the mathematical function of multiobjective topology optimization of joints that considers both stiffness and frequency can be obtained by the compromise programming method:

$$
\left\{\begin{array}{l}
\text { find } \boldsymbol{\rho}=\left\{\boldsymbol{\rho}_{1}, \boldsymbol{\rho}_{2}, \ldots, \boldsymbol{\rho}_{n}\right\}^{T}, \\
\min F(\boldsymbol{\rho})=\left\{\alpha^{P}\left[\sum_{k=1}^{m} \omega_{k}^{P} \frac{C_{k}(\boldsymbol{\rho})-C_{k}^{\min }}{C_{k}^{\max }-C_{k}^{\min }}\right]^{p}+(1-\alpha)^{P}\left(\frac{\Lambda_{\max }-\Lambda(\boldsymbol{\rho})}{\Lambda_{\max }-\Lambda_{\min }}\right)^{P} \frac{1}{P},\right. \\
\frac{\text { s.t. } V(\boldsymbol{\rho})}{V_{0} \leq f} \mathbf{K}(\boldsymbol{\rho}) \mathbf{U}_{k}=\mathbf{F}_{k}, k=1,2, \ldots, m, \\
\left(\mathbf{K}(\boldsymbol{\rho})-\lambda_{r} \mathbf{M}(\boldsymbol{\rho})\right) \beta_{r}=0, r=1,2, \ldots, D_{e},
\end{array}\right.
$$


where $F(\rho)$ is the objective function of multiobjective optimization, $\alpha$ is the weight of the static stiffness objective, and $\Lambda_{\max }$ and $\Lambda_{\min }$ are the maximum and minimum values of the objective function during frequency optimization, respectively.

3.4. Weight of Each Load Condition. The weight $\omega_{k}$ corresponding to each static condition is required when using the compromise programming method to solve multiobjective topology optimization problems, the traditional method generally used the experience to assign this variable, but this led to the following shortcomings:

(1) The weight value given is only ascertained based on the designer's experience and is not accurate.

(2) The designer is required to have a wealth of engineering experience, which increases the difficulty of design.

(3) The accurate weight of various load conditions cannot be given when there are several operating conditions.

To solve the above problems, the AHP [28] is used to determine the weight $\omega_{k}$ of each load condition. The AHP is a method that decomposes the elements related to decisionmaking into goals, criteria, plans, and other levels, performing qualitative and quantitative analysis on this basis. The method is as follows: first, establish the decision-making level of the problem to be evaluated, adopt the 1-9 scale method, and construct the judgment matrix $\boldsymbol{A}=\left(\boldsymbol{A}_{i j}\right)_{m \times m}$ $\left(\boldsymbol{A}_{i j}\right.$ is the degree of importance of load condition $i$ relative to load condition $j$, which is assigned by the designer according to the relative importance shown in Table 1). Then, use the eigenvector method to calculate the eigenvalue and eigenvector of $\boldsymbol{A}$ and normalize the eigenvector corresponding to the largest eigenvalue to the weight coefficient vector $\omega$.

For matrix $\boldsymbol{A}$, when the number of eigenvectors corresponding to a positive eigenvalue is more than 1 , it is necessary to further determine which eigenvector represents the required weight vector. Therefore, a prerequisite is to ensure the consistency of the judgment matrix. If the consistency requirements are not met, the judgment matrix needs to be rebuilt. According to the judgment matrix consistency criterion defined in a previous study [28], each pairing matrix can use a consistency ratio $(C R)$ to ascertain the degree of matrix consistency. If the consistency ratio satisfies the following formula, the judgment matrix is reasonable. Otherwise, it needs to be redefined:

$$
\begin{aligned}
& C R=\frac{C I}{R I}<0.10, \\
& C I=\frac{\beta_{\max }-m}{m-1},
\end{aligned}
$$

where $C I$ is the consistency index, $m$ is the number of load conditions, $\beta_{\max }$ is the maximum eigenvalue of the matrix $\boldsymbol{A}$, and $R I$ is the average random consistency index, whose value can be found in Table 2 .
Table 1: Definition of importance scale of judgment matrix $\boldsymbol{A}$.

\begin{tabular}{lc}
\hline Scaling $\left(\mathrm{a}_{i j}\right)$ & Importance \\
\hline 1 & $i$ and $j$ are equally important \\
3 & $i$ is slightly more important than $j$ \\
5 & $i$ is obviously more important than $j$ \\
7 & $i$ is far more important than $j$ \\
9 & $i$ is significantly more important than $j$ \\
$1 / 3$ & $j$ and $i$ are equally important \\
$1 / 5$ & $j$ is obviously more important than $i$ \\
$1 / 7$ & $j$ is far more important than $i$ \\
$1 / 9$ & $j$ is significantly more important than $i$ \\
$2,4,6,8$ & The middle of each scale \\
\hline
\end{tabular}

TABle 2: Average random consistency index.

\begin{tabular}{ccccccccccc}
\hline$m$ & 1 & 2 & 3 & 4 & 5 & 6 & 7 & 8 & 9 & 10 \\
$R I$ & 0 & 0 & 0.58 & 0.90 & 1.12 & 1.24 & 1.32 & 1.41 & 1.45 & 1.49 \\
\hline
\end{tabular}

Assuming that the vector $\psi=\left(\psi_{1}, \psi_{2}, \ldots, \psi_{m}\right)$ is the eigenvector corresponding to the maximum eigenvalue $\beta_{\max }$ of matrix $A$ when matrix $A$ satisfies equation (6), the weight of the $k$-th load condition can be calculated as follows:

$$
\omega_{k}=\frac{\psi_{k}}{\psi_{1}+\psi_{2}+\cdots+\psi_{m}},
$$

where $\psi_{k}$ is the $k$-th value in the eigenvector.

3.5. Multiobjective Topology Optimization Process. Based on the OptiStruct optimization program, the method of moving asymptotes is used as the optimization algorithm, and the lower-limit constraint and the perimeter constraint are used to control numerical instability. The compromise programming method is used to optimize the functions of the static stiffness and dynamic frequency. The optimized function is combined into a comprehensive objective function, and used in conjunction with the AHP to determine the weight of each load condition of the subobjective. Taking the volume fraction as the constraint and the minimization of the comprehensive objective function as the goal, the joint is optimized for multiobjective topology. The optimization process is shown in Figure 1.

According to Figure 1, in the process of joint optimization, the joint model is first established, and then the model is divided into finite elements and boundary conditions are imposed. Then, the joint is calculated by singleobjective optimization, the optimal result obtained by singleobjective optimization is brought into the multiobjective optimization function, and the joint is solved by multiobjective optimization by applying constraints, responses, and goals. Finally, the rationality of multiobjective optimization is analyzed. If the result is reasonable, the optimized structure is smoothed, and a model with a reasonable structure is obtained. Otherwise, it should be redesigned.

\section{Topology Optimization of Spherical Spatial Grid Structure Joints}

4.1. Finite Element Models of Spherical Joints. As shown in Figure 2(a), a joint with a radius of $300 \mathrm{~mm}$, which has a 
design area in the shape of a solid sphere is used, where the eight circumscribed pipes are nondesigned areas. The outer radius of the extension pipe is $90 \mathrm{~mm}$, the inner radius is $70 \mathrm{~mm}$, and the outer elongation is $95 \mathrm{~mm}$. Four of the pipes are located in the middle hemisphere, and the extension directions are along the $x$ - and $y$-axes. The other four are located in the upper hemisphere, and the absolute value of the angle between their directions and the $x, y$, and $z$ axes is 45 degrees upward. The joint meshing uses tetrahedral elements with an average size of $10 \mathrm{~mm}$, forming a total of 87398 nodes and 457303 elements. The material is Q345 steel, whose yield strength is $345 \mathrm{MPa}$, the elastic modulus is $210 \mathrm{GPa}$, Poisson's ratio is 0.3 , and density is $7800 \mathrm{~kg} / \mathrm{m}^{3}$.

The leftmost overhanging pipe is the fixed end, and the axial and shear load on each of the remaining seven tubes is $124.8 \mathrm{kN}$. Axial force $(\mathrm{N})$ represents the axial tension in the middle four tubes and the axial pressure in the upper four tubes, the shear force $(Q)$ is along the $z$-axis direction in the middle four tubes, is perpendicular to the plane normal direction on the upper four tubes, and is inclined upward at an angle of 45 degrees with the adjacent two tubes. The bending moment load $(M)$ on each tube is $40 \mathrm{kN} \mathrm{m}$, and its direction is clockwise along with the shear load, as shown in Figure 1. The total steel consumption of the joint before the optimization is $960.5 \mathrm{~kg}$. The figure with loads direction and boundary conditions is shown in Figure 2(b).

4.2. Determination of the Weight of Each Load Condition. In this paper, the joint action of axial, shear, and bending moment loads is considered according to the relative importance degree of each working condition. The axial load is obviously more important than the shear and bending moment loads, and the shear load is slightly more important than the bending moment load, according to Table 1. The judgment matrix $\boldsymbol{A}$ is built. Using the AHP to obtain the weight proportion of each working condition, and the constructed judgment matrix is as follows:

$$
\mathbf{A}=\left(\begin{array}{ccc}
1 & 5 & 7 \\
\frac{1}{5} & 1 & 3 \\
\frac{1}{7} & \frac{1}{3} & 1
\end{array}\right) .
$$

The maximum eigenvalue is found to be 3.06489 , and the corresponding eigenvector $\psi=(0.96280,0.24826,0.10669)^{\mathrm{T}}$. By substituting the eigenvalue and $R I=0.58$-found in Table 2 into equation (6), the $C R$ can be obtained as follows:

$$
C R=\frac{C I}{R I}=\frac{3.06489-3}{2 * 0.58}=0.0559<0.10 .
$$

Equation (10) satisfies the requirements of equation (6). That is, the judgment matrix $\boldsymbol{A}$ is reasonably constructed, and the weights $\omega_{1}=0.731, \omega_{2}=0.188$, and $\omega_{3}=0.091 \mathrm{can}$ be obtained from equation (8). Therefore, the weight coefficients of the axial, shear, and bending moment loads are $0.731,0.188$, and 0.081 , respectively.

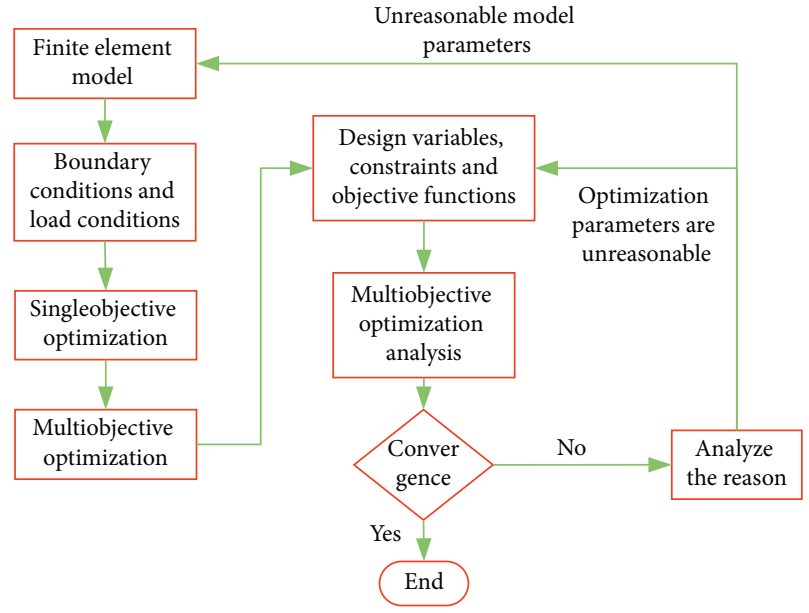

FIGURE 1: Multiobjective topology optimization process.

4.3. Topology Optimization of Spherical Joints under Static Conditions. For the topology optimization of spherical joints under static conditions, the objective function must minimize compliance (i.e., maximize stiffness). Further, the $40 \%$ volume fraction is a constraint, the penalty factor is set to 2 , and the minimum member size is taken as 2.5 times the unit size, i.e., $25 \mathrm{~mm}$. The stiffness models under different conditions are analyzed for topology optimization, and the iterative curves of compliance under different conditions are shown in Figure 3.

Figure 3 shows that the iterative convergence steps are 50,50 , and 48 , respectively, under the action of axial, shear, and bending moment loads. With the increase in the number of iterative steps, the compliance corresponding to each working condition gradually decreases and finally converges. The maximum compliance and minimum compliance of the structure corresponding to each working condition are shown in Table 3.

The maximum and minimum compliance in Table 3 are $C_{k}^{\max }$ and $C_{k}^{\min }$, respectively, corresponding to equation (5) of the multiobjective optimization mathematical function.

\subsection{Structural Dynamic Topology Optimization Aiming at} Maximizing Low-Order Frequency. When performing topology optimization on the spherical joint shown in Figure 2, the first three-order frequencies are selected as the research object and optimized according to the objective function defined in equation (4). For the joint, the low-order frequencies account for a larger proportion of the total, and the weights assigned to the first three-order frequencies are $0.5,0.3$, and 0.2 [29]. The design variables and constraints are the same as those used in static optimization. The maximum average eigenvalue is used as the optimization goal. The change curve of the first three natural frequencies in the optimization process is shown in Figure 4.

It can be seen from Figure 4 that the optimization converges at the 48th iteration step. The first three natural frequencies exhibited an overall upward trend during the optimization process, with no oscillation phenomenon, and the change trends of the first three frequencies were the 


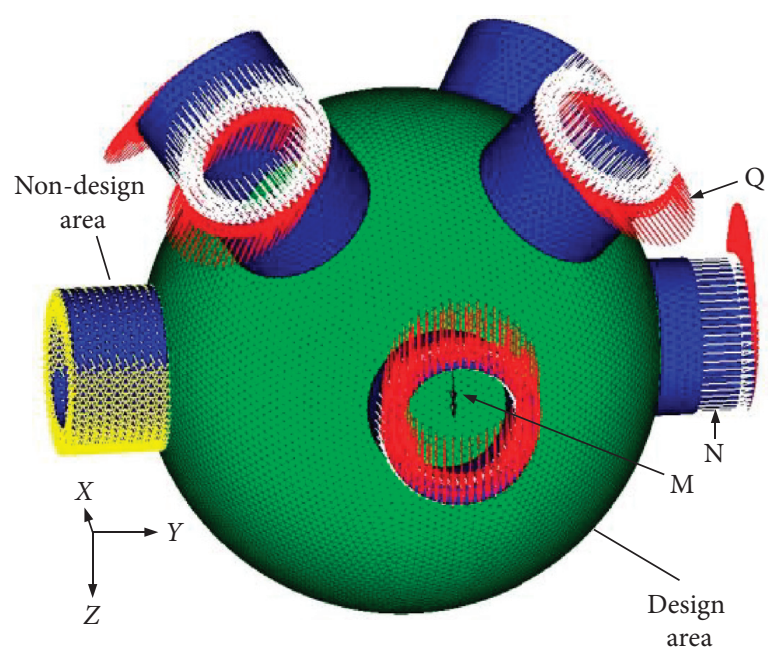

(a)

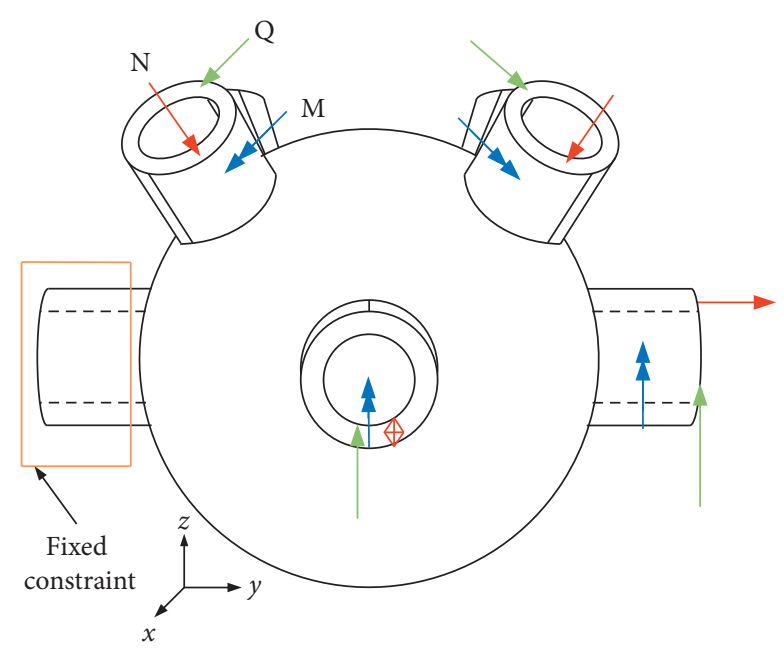

(b)

Figure 2: Finite element model and load arrangement of the joint. (a) Finite model. (b) Loads and boundary conditions.

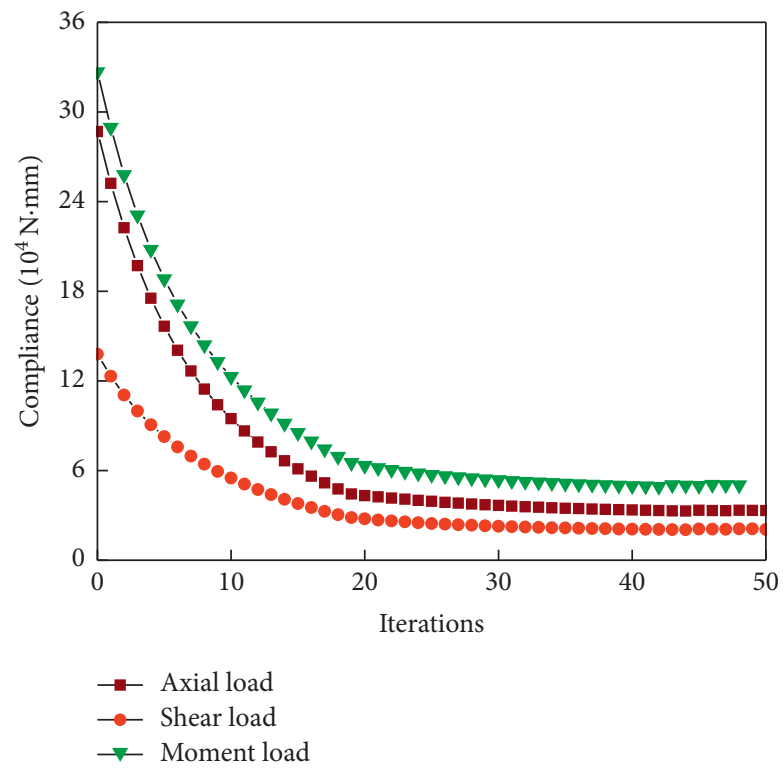

Figure 3: Iteration step-compliance curve under various load conditions.

TABle 3: Maximum and minimum compliance under various load conditions.

\begin{tabular}{lcr}
\hline Conditions & Max compliance $/ 10^{4} \mathrm{~N} \cdot \mathrm{mm}$ & Min compliance $/ 10^{4} \mathrm{~N} \cdot \mathrm{mm}$ \\
\hline Axial & 28.68 & 3.29 \\
Shear & 13.80 & 2.04 \\
Bending moment & 32.68 & 4.93 \\
\hline
\end{tabular}

same. The description of the maximum and minimum frequencies, along with the mode formation during the optimization process, is given in Table 4.

By substituting the maximum and minimum frequencies shown in Table 4 into equation (4), the maximum and minimum values of $\Lambda(\rho)$ in the frequency optimization can be obtained, that is, the $\Lambda_{\max }$ and $\Lambda_{\min }$ of equation (5) in multiobjective topology optimization.
4.5. Multiobjective Topology Optimization of Spherical Joints. In the multiobjective topology optimization study, the weight coefficient of each load condition is obtained according to equation (9). To obtain the comprehensive objective of topology optimization, according to previous literature [25], the weights of compliance and frequency are 0.6 and 0.4 , respectively. Further, and the maximum and minimum compliance corresponding to each working 


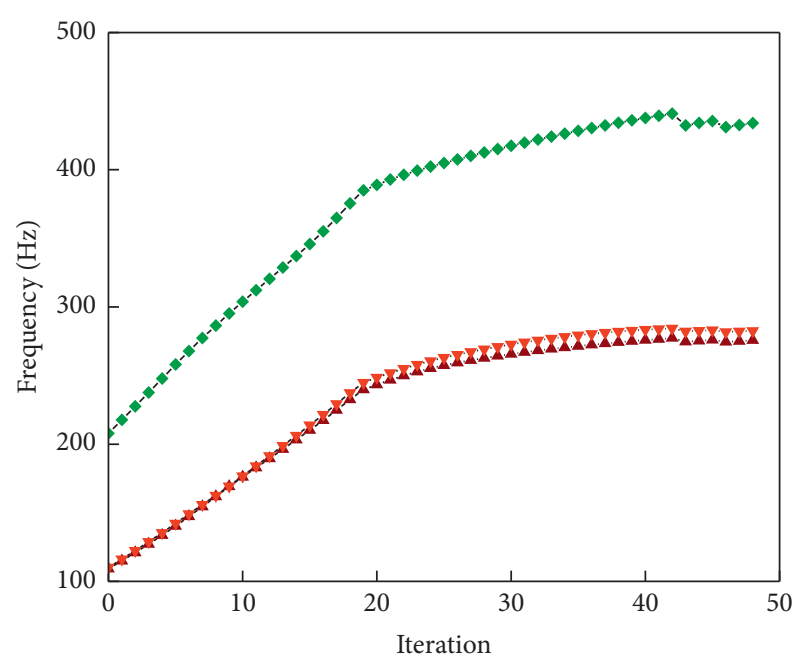

$$
\begin{aligned}
& \text { Frequency } \\
& \rightarrow-1 \text { th } \\
& \rightarrow-2 \text { th } \\
& \rightarrow-3 \text { th }
\end{aligned}
$$

FIgURE 4: Variation curve of the first three natural frequencies in the optimization process.

TABle 4: Maximum and minimum frequencies and modes in dynamic frequency optimization.

\begin{tabular}{lccc}
\hline Order & $\begin{array}{c}\text { Max frequency/ } \\
\mathrm{Hz}\end{array}$ & $\begin{array}{c}\text { Min frequency/ } \\
\mathrm{Hz}\end{array}$ & Formation \\
\hline 1 & 277.33 & 109.19 & $\begin{array}{c}\text { Along the }-y- \\
\text { direction } \\
\text { Along the }-z- \\
\text { direction }\end{array}$ \\
3 & 284.40 & 110.16 & $\begin{array}{c}\text { Twisting along the } x \text { - } \\
\text { axis }\end{array}$ \\
\hline
\end{tabular}

condition shown in Table 3 and the eigenvalues corresponding to the maximum and minimum frequencies, shown in Table 4, are substituted into the multiobjective optimization objective function in equation (5), with the same restrictions as those in single-objective optimization. The optimization objective is to minimize the multiobjective function, and the change curve of the objective function during the optimization process is shown in Figure 5.

It can be seen from Figure 5 that the value of the objective function, defined according to the compromise programming method, gradually decreases and converges at the 22 nd iteration step. At step 0 , the value of the objective function is 0.575 , and its value is 0.481 at the 22 nd step. For multiobjective optimization, the change curve of the structural compliance and frequency under each load condition throughout the iteration process is shown in Figure 6.

Figure 6(a) shows that the change in the compliance trend corresponding to the three working conditions is the same, and the optimized compliances all have different levels of decline and convergence. Figure 6(b) shows the frequency iteration process of multiobjective optimization. It can be

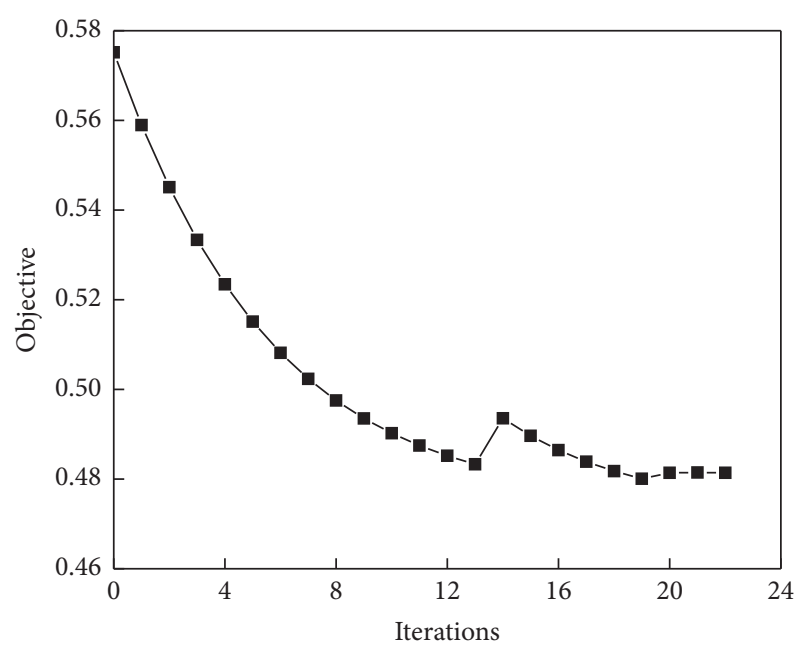

Figure 5: Change curve of the objective function in the optimization process.

seen that the first three frequencies have improved to varying degrees, the fluctuation trend is the same, and there is no oscillation caused by frequency alternation.

Table 5 shows the specific values of the initial compliance and optimized compliance. The compliance values corresponding to the three working conditions have been reduced from 21.49, 10.35, and 24.87 to $7.11,6.00$, and 15.45 $\left(10^{4} \mathrm{Nmm}\right)$. This shows that multiobjective topology optimization significantly improves structural rigidity.

Table 6 shows the frequency changes before and after optimization. The first three-order frequencies are increased from 109.19, 110.16, and 207.89 to $166.98,185.11$, and 289.89 $(\mathrm{Hz})$, respectively, which represent a significant increase. This indicates that multiobjective topology optimization has significantly improved the dynamic performance of the structure.

The density distribution of the joint obtained after optimization is shown in Figure 7, and the structure satisfying both static multiload stiffness and dynamic natural frequency objectives is obtained, with clear stress transmission paths. The two adjacent pipes in the upper hemisphere are connected by thin rods, and the two adjacent pipes in the lower hemisphere are connected by thick pipes. Several symmetrical holes are formed in the middle of the pipe, the joint form is novel, its overall stability is strong, and the structure has no directionality, which meets the design requirements.

\section{Analysis of the Results}

5.1. Extraction of Optimized Results. To determine the final optimized shape, the topology optimization results are further processed according to the density threshold. The density threshold controls the selection of the minimum density after the optimization of the structure, with a value between 0 and 1 . The selection of the density threshold is crucial to determine the final structural shape. 


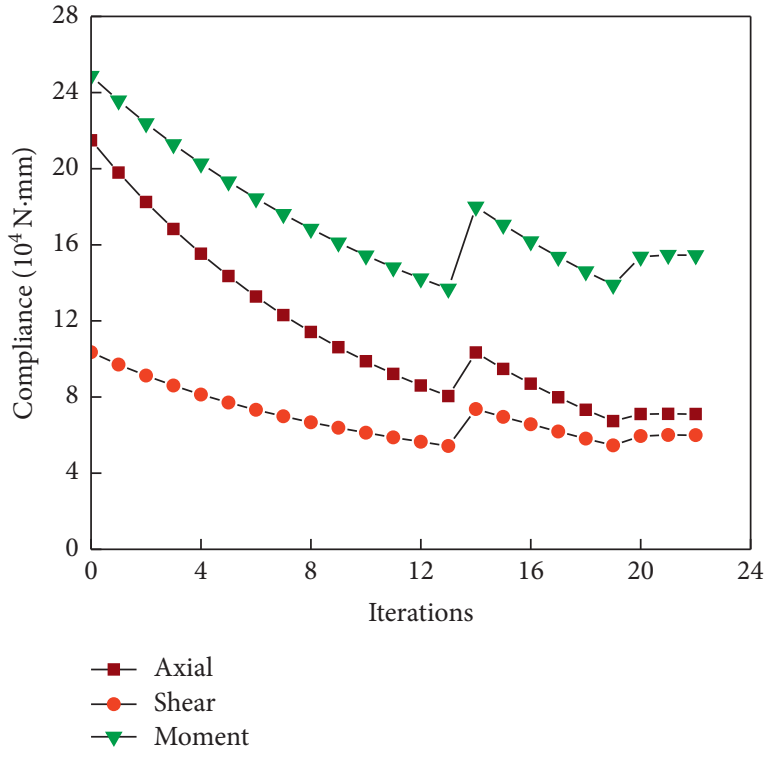

(a)

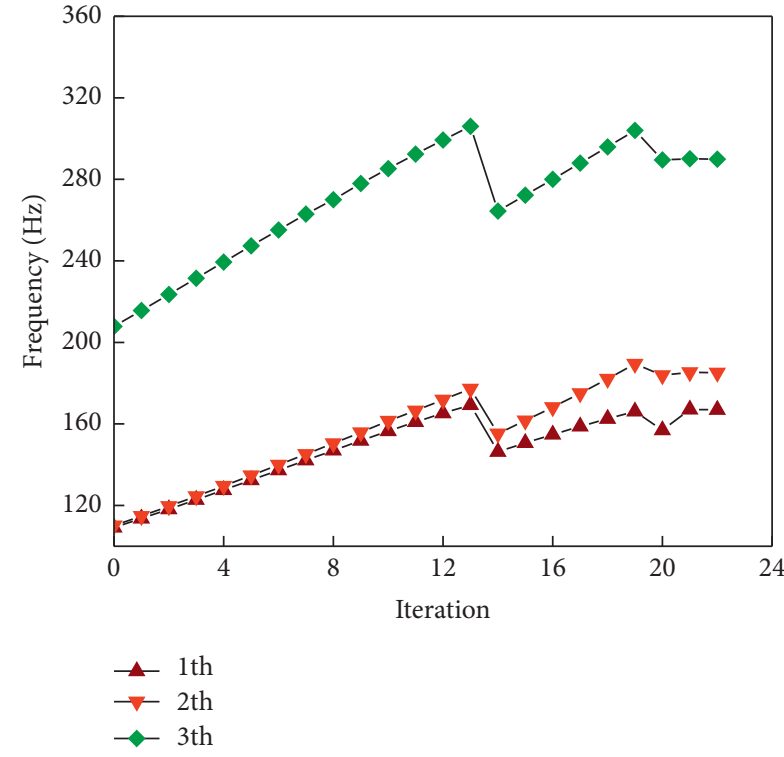

(b)

Figure 6: Compliance and frequency variation curve in the optimization process. (a) Compliance variation curve. (b) Frequency variation curve.

TABLE 5: Initial compliance and optimized compliance

\begin{tabular}{lcr}
\hline Load conditions & Initial compliance $\left(10^{4} \mathrm{~N} \cdot \mathrm{mm}\right)$ & Optimized compliance $\left(10^{4} \mathrm{~N} \cdot \mathrm{mm}\right)$ \\
\hline Axial & 21.49 & 7.11 \\
Shear & 10.35 & 6.00 \\
Bending moment & 24.87 & 15.45 \\
\hline
\end{tabular}

TABLE 6: Description of frequency before and after optimization and formation.

\begin{tabular}{|c|c|c|c|}
\hline Order & Initial frequency $(\mathrm{Hz})$ & Optimized frequency $(\mathrm{Hz})$ & Formation \\
\hline First & 109.19 & 166.98 & Along the $-y$-direction \\
\hline Second & 110.16 & 185.11 & Along the $-z$-direction \\
\hline Third & 207.89 & 289.89 & Twisting along the $x$-axis \\
\hline
\end{tabular}

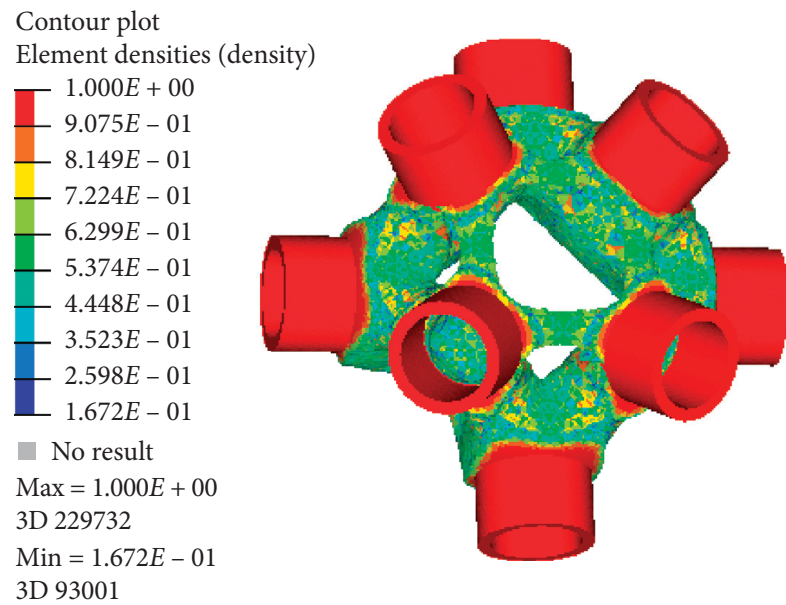

Figure 7: Density distribution of the optimized joint.
According to the "Code for Design of Steel Structures" GB 50017-2017, for cast steel joints, the stress under complex loads should be less than $1.1 \mathrm{fy}$, that is, less than $379.5 \mathrm{MPa}$. It can be seen from Figure 8 that, when $T$ is 0.8 , the maximum von Mises stress of the structure under axial, shear, and bending moment loads reaches $827.5 \mathrm{MPa}, 906.5 \mathrm{MPa}$, and $740.1 \mathrm{MPa}$, respectively. Further, the maximum stress in the structure under the combined working conditions also far exceeds the stress of the structure when the threshold is 0.7. The stress in the structure is highly concentrated. At this time, the structural material has yielded, so the upper limit of the density threshold should be taken as a value less than 0.8 . When the threshold value is $0.2-0.7$, the steel consumption and change rate corresponding to different threshold values are shown in Table 7.

Table 7 shows that, as the threshold increases, the amount of steel used in the joint gradually decreases. To obtain a clearer relationship between the mechanical 


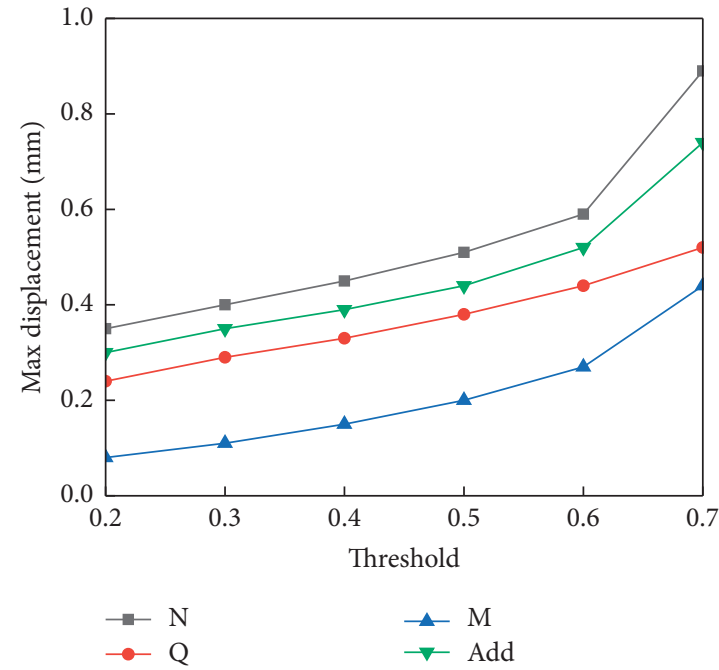

(a)

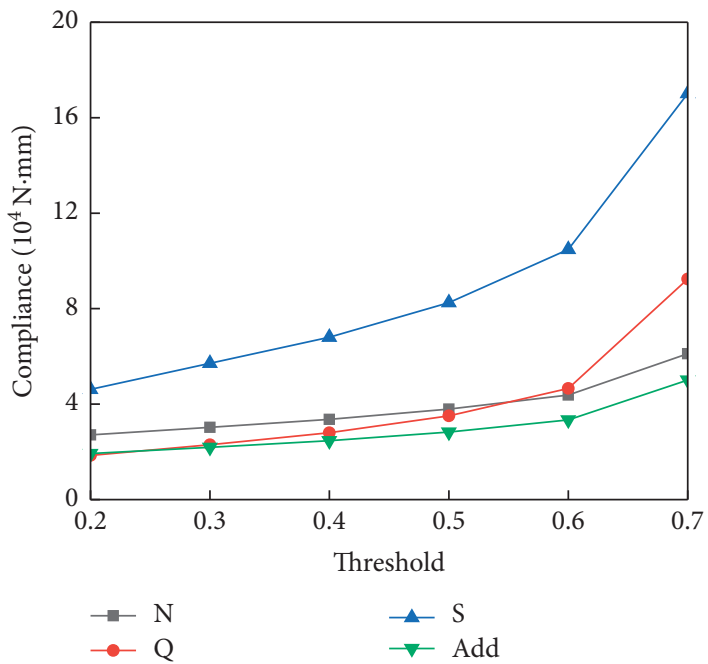

(c)

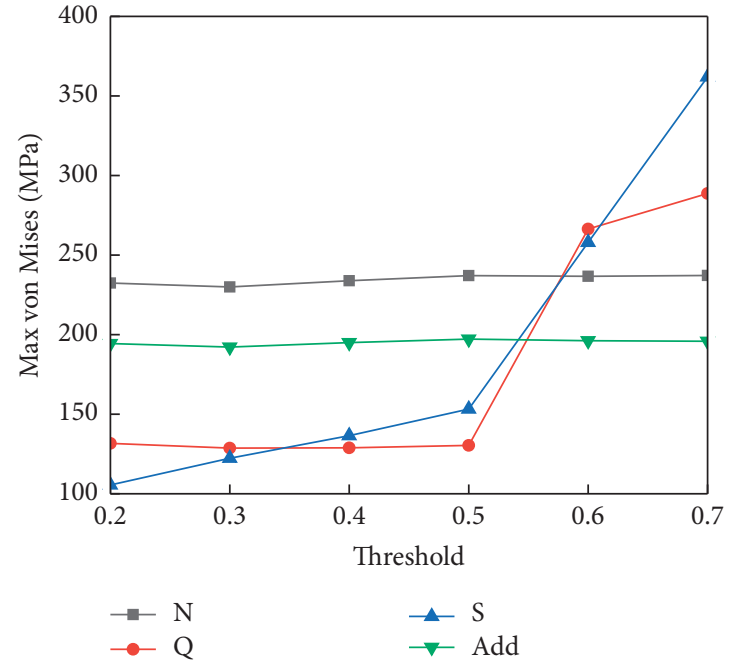

(b)

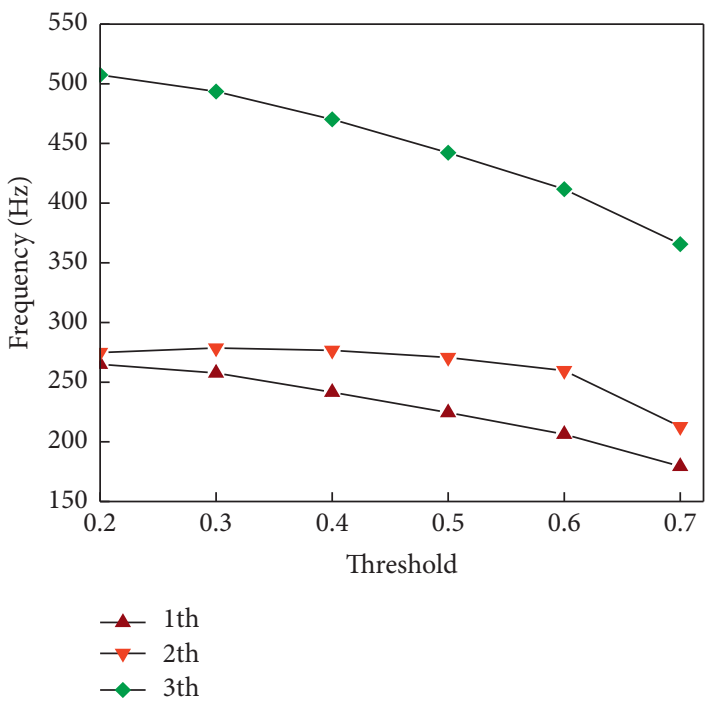

(d)

FIGURE 8: Mechanical property curves corresponding to different thresholds. (a) Maximum displacement. (b) von Mises stress. (c) Compliance. (d) Frequency.

TABLE 7: Steel consumption and change rate at different thresholds.

\begin{tabular}{lcccccc}
\hline $\mathrm{T}$ & 0.2 & 0.3 & 0.4 & 0.5 & 0.6 & 0.7 \\
\hline $\begin{array}{l}\text { Steel } \\
\text { consumption/kg }\end{array}$ & 46.78 & 37.79 & 32.43 & 28.24 & 24.54 & 20.59 \\
Change rate\% & & -19.22 & -14.18 & -12.92 & -13.10 & -16.10 \\
\hline
\end{tabular}

properties corresponding to different thresholds, according to Figure 8, the maximum displacement of the structure, maximum von Mises stress, compliance, and frequency with the threshold change curve are shown in Figure 8.

Figure 8(a) shows the maximum displacement of the structure at different thresholds. It can be seen that, with increases in the density threshold, the maximum displacement of the structure under the action of the bending moment gradually becomes larger. When the threshold exceeds 0.6, the axial, bending moment, and structural displacement increase significantly under the combined working conditions, and the structural resistance to displacement is significantly reduced at this time.

It can be seen in Figure 8(b) that, with increases in the density threshold, the von Mises stress changes only slightly under the action of axial load and combined load conditions, but when the threshold exceeds 0.5 , the von Mises stress of the structure increases rapidly under the action of shear and bending moment loads. At this time, there is a large stress concentration in the structure.

It can be seen from Figure 8(c) that, when the threshold is less than 0.6 , as the threshold value increases, the respective functions of the three conditions and the corresponding compliance of the combined conditions gradually increase. When the threshold is higher than 0.6, the compliance under shear and bending moment conditions increases, and the structural rigidity decreases significantly. 
The structural compliance is low under combined working conditions, which demonstrates that the structure has better performance in resisting various loads.

Figure 8(d) shows the first three-order frequency change curve. As the threshold value increases, the frequency slowly decreases.

According to the above analysis, it is clear that when the density threshold is 0.5 , the steel consumption of the optimized joint will be greatly reduced, and the structural performance is better. To obtain a topological form with better mechanical properties, the density threshold is taken as 0.5 , and the corresponding structural, topological density distribution is shown in Figure 9.

It can be seen that the optimized structural topology is bidirectionally symmetrical along the central axis, with a large hole in the middle and a small hole on each side. The structure does not have directionality. Figure 9(b) shows a cross-sectional view of the structure. It can be seen that the materials on the left and right sides are symmetrically arranged, and most of the materials in the lower half of the sphere have been deleted. From the three-dimensional view in Figure 9(c), it can be seen that there are many holes in the upper hemisphere. Further, the adjacent two pipes are connected by a connecting rod, with clear force transmission, forming a reasonable structure.

5.2. Smoothing of the Optimized Results. As shown in Figure 10(a), the optimized structure is more complex and has many corners. To obtain a smoother structure, Altair solidThinking Evolve is used to smooth it. The topological form of the processed joint is shown in Figure 10(b). It can be seen that, in comparison to the preliminary optimized structure, the smoothed joint has no sharp corners, a continuous connection of various parts, and a more aesthetic shape.

5.3. Comparison of Mechanical Performance. For spatial grid structures, hollow sphere joints are commonly used. To verify the performance of the optimized joints, a welded hollow sphere joint is created. The thickness of the hollow sphere is $36.5 \mathrm{~mm}$, and the material properties of both joints are the same. The steel consumption and mechanical properties of the two joints are compared in Table 8.

Table 8 shows that the difference in steel consumption between the hollow ball joint and the optimized joint is only $0.3 \%$. Under the bending moment condition, the maximum displacement of the optimized joint is $17.6 \%$ larger than that of the hollow spherical joint, but the corresponding stress under the bending moment condition is reduced by $34.7 \%$, and the structural stress distribution is more uniform. Under other load conditions and the combined load condition, the optimized joint exhibits a larger reduction in stress and displacement, and the mechanical performance is significantly better than that of the hollow spherical joint. This is because the weight of the bending moment condition only accounts for $8.1 \%$ in the multiobjective topology optimization. The optimized design can improve the mechanical properties of the structure under the axial and shear conditions, which is in line with the multiobjective optimization design, whose principle is to allocate materials reasonably according to different weights of the load conditions.

Through a comparison of the change rate of performance under the action of the three load conditions, it can be seen that the change rate of stress and displacement of the optimized joint under the action of axial, shear, and bending moment loads are $-37.8 \%$ and $-50.0 \%,-44.2 \%$ and $-34.9 \%$, and $-34.7 \%$ and $17.6 \%$, respectively, and the structural stress and displacement change rates under combined conditions are $-37.9 \%$ and $46.5 \%$, respectively. For spatial grid structures, the main mode of loading is axial loading. Through topology optimization, the weight of the axial load obtained using the AHP is 0.731 . Through the process of topology optimization design, more materials are allocated to bear the axial load. The obtained joint can resist axial forces significantly better than it can resist shear forces and bending moments. The 1st frequency of the optimized joint is increased by $34.4 \%$. In comparison to the traditional hollow spherical joint, the optimized joint has a more uniform stress distribution and greater rigidity.

Based on the above analysis, it can be concluded that in comparison to the hollow spherical joint, the joint designed through topology optimization has a better static and dynamic performance. Compared with the traditional singleobjective topology optimization design, multiobjective optimization is more suitable for engineering practice since it considers both static and dynamic design objectives.

The most widely used casting method for the optimized joint is additive manufacturing [30], which greatly reduces the time and labor cost through the layer-by-layer stacking of materials. The principle of the printing process is as follows:

(1) Import the STereoLithography (STL) format file into the $3 \mathrm{D}$ printer, design the scanning path, and control the movement of the laser scanner and lifting platform through the generated data;

(2) A laser beam irradiates the surface of the liquid photosensitive resin through the scanner controlled by a numerical control device according to the designed scanning path so that a layer of resin is solidified in the specific area of the surface. When processing of one layer is completed, the first section of the part is generated.

(3) The lifting platform is lowered a certain distance and a new layer of liquid resin covers the cured layer. The second layer is scanned, and then the second cured layer is firmly bonded to the previously cured layer. In this way, a complete $3 \mathrm{D}$ workpiece prototype was formed through layer-by-layer superposition.

(4) Finally, the prototypes are taken out of the resin and cured.

However, currently, the metal powder required for additive manufacturing is relatively expensive. To reduce this 


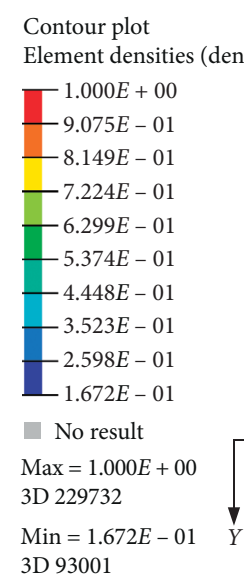
3D 93001

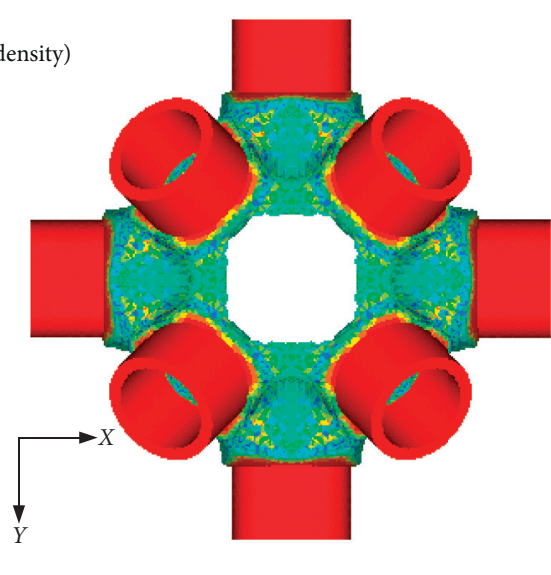

(a)

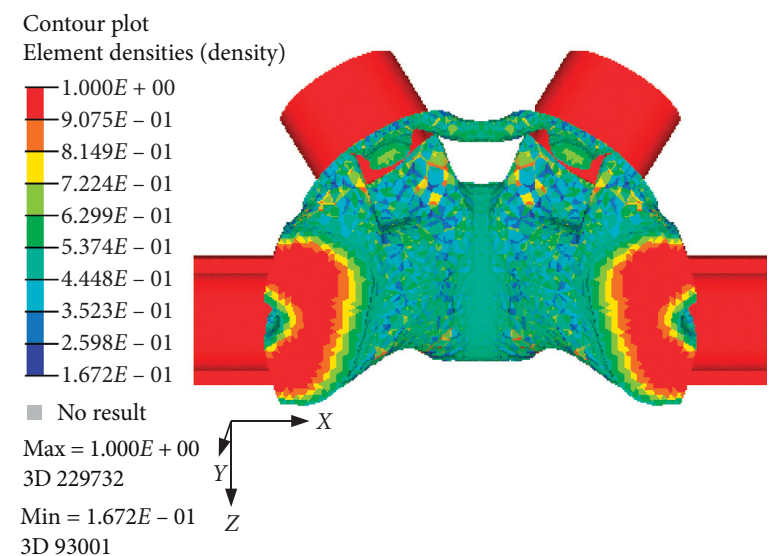

(b)

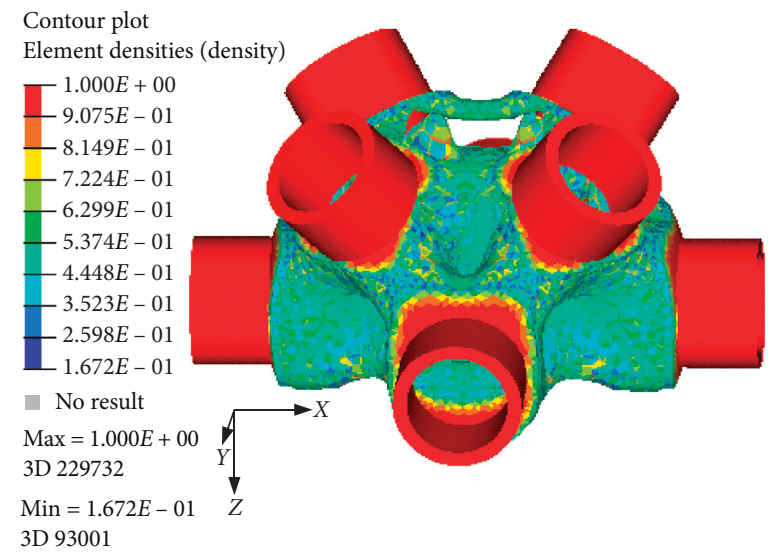

(c)

Figure 9: Topological density distribution of spherical joints with a threshold value of 0.6. (a) Top view. (b) Sectional half-structure view. (c) Three-dimensional view.

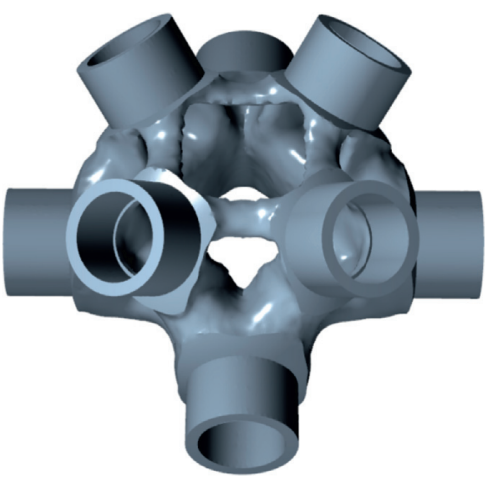

(a)

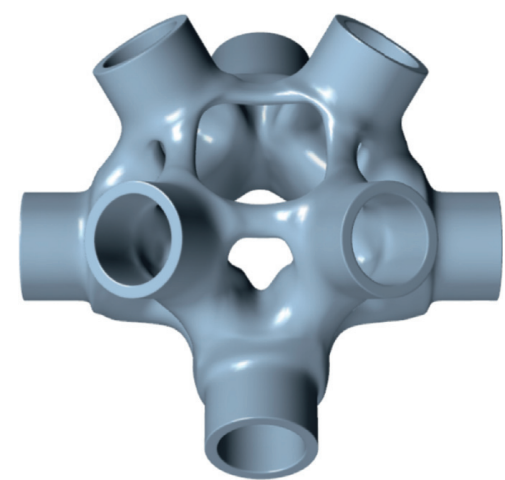

(b)

FIgURE 10: Optimized and Smoothed joint. (a) Initial optimization. (b) Smoothed optimization.

cost, researchers have proposed some methods to combine additive manufacturing with traditional castings, such as EPC-sand casting technology [31], through the 3D printing of cheap sand and foam, which is then poured into a closed box. Wax mold casting technology, by printing wax molds, hanging paste to form molds for casting. With developments in technology, the cost of additive manufacturing will gradually decrease, and the industrial production of spatialstructure topology optimization nodes will soon be realized [32]. 
TABLE 8: Comparison of the joints' performance.

\begin{tabular}{lccc}
\hline Parameters & Hollow joint & Optimized joint & Difference\% \\
\hline Weight/kg & 358.6 & 359.8 & 0.3 \\
Axial condition stress/MPa & 445.0 & 276.6 & -37.8 \\
Axial condition displacement/mm & 1.12 & 0.56 & -50.0 \\
Shear condition stress/MPa & 259.0 & 144.4 & -44.2 \\
Shear condition displacement/mm & 0.63 & 0.41 & -34.9 \\
Bending moment condition stress/MPa & 224.3 & 0.20 & -34.7 \\
Bending moment condition displacement/mm & 0.17 & 229.4 & 17.6 \\
Combined condition stress/MPa & 369.6 & 0.407 & -37.9 \\
Combined condition displacement/mm & 0.936 & 213.91 & -46.5 \\
lst frequency/Hz & 159.13 & $34.4 \%$ \\
\hline
\end{tabular}

\section{Conclusion}

In this study, the multiobjective topology optimization method was applied to the topology optimization of the design of spatial-structure joints, and a design scheme that satisfied the minimum requisite compliance and maximized the low-order frequency was proposed. The main conclusions are as follows:

(1) The compromise programming method is applied to the multiobjective topology optimization of spatial spherical joints, which comprehensively considers the multiobjective optimization of stiffness and frequency under static axial, shear, and bending moment loads, introducing the analytic hierarchy process to obtain the weight coefficient of load conditions. This mitigates the limitation of considering a single objective in the traditional topology optimization of joints and the empirical value of load conditions.

(2) Minimizing the compliance and maximizing the first three-order frequencies to construct a multiobjective optimization function is comprehensively analyzed, taking the volume fraction as the constraint. By conducting multiobjective topology optimization for the joints, a new topology for the joint is obtained, which conforms to the load transmission path. The optimized joint has high static and dynamic performance and mitigates the oscillation problem caused by frequency alternation.

(3) The optimized structure is smoothed to obtain an optimized joint with reasonable stress properties and improved aesthetics. The mechanical properties of the optimized joint and that of the welded hollow sphere joint are compared. In comparison to the hollow sphere joint, the optimized joint has better static and dynamic performance and uses the same amount of steel. In comparison to the traditional single-objective topology optimization design, multiobjective optimization is more suitable for engineering applications. The rationality of multiobjective topology optimization is verified, and an effective solution is provided for the lightweight design of joints.

\section{Data Availability}

The data used to support the findings of this study are available from the corresponding author upon request.

\section{Conflicts of Interest}

The authors declare that they have no conflicts of interest.

\section{Acknowledgments}

This work described in this paper has been funded in part by the National Natural Science Foundation of China (No. 51768024).

\section{References}

[1] C. Ding, H. Seifi, S. Dong, and Y. M. Xie, "A new nodeshifting method for shape optimization of reticulated spatial structures," Engineering Structures, vol. 152, no. 12, pp. 727-735, 2017.

[2] H. Lin, Y. Zhu, J. Yang, Z. Wen, and H. B. Ozmen, "Anchor stress and deformation of the bolted joint under shearing," Advances in Civil Engineering, vol. 2020, Article ID 3696489, 10 pages, 2020.

[3] J. Bai, G. Meng, H. Wu, and W. Zuo, "Bending collapse of dual rectangle thin-walled tubes for conceptual design," ThinWalled Structures, vol. 135, pp. 185-195, 2019.

[4] J. Bai, G. Meng, W. Zuo, and W. J. Zuo, "Rollover crashworthiness analysis and optimization of bus frame for conceptual design," Journal of Mechanical Science and Technology, vol. 33, no. 7, pp. 3363-3373, 2019.

[5] C. Gui, J. Bai, and W. Zuo, "Simplified crashworthiness method of automotive frame for conceptual design," ThinWalled Structures, vol. 131, pp. 324-335, 2018.

[6] H. Seifi, M. Xie, J. O’Donnell, and N. Williams, "Design and fabrication of structural connections using Bi-directional evolutionary structural optimization and additive manufacturing," Applied Mechanics and Materials, vol. 846, pp. 571-576, 2016.

[7] N. Changizi and G. P. Warn, "Stochastic stress-based topology optimization of structural frames based upon the second deviatoric stress invariant," Engineering Structures, vol. 224, Article ID 111186, 2020.

[8] N. Shinji and T. Kenjiro, "Advanced topology optimization," International Journal for Numerical Methods in Engineering, vol. 113, no. 08, pp. 1145-1147, 2018. 
[9] T. Zegard, C. Hartz, A. Mazurek, and W. F. Baker, "Advancing building engineering through structural and topology optimization," Structural and Multidisciplinary Optimization, vol. 62, no. 2, pp. 915-935, 2020.

[10] M. C. Chen, Y. Zhao, and Y. M. Xie, "Topology optimization and additive manufacturing of nodes in spatial structures," China Civil Engineering Journal, vol. 52, no. 02, pp. 1-10, 2019.

[11] L. Li and X. Zhu, "Design of compliant revolute joints based on mechanism stiffness matrix through topology optimization using a parameterization level set method," Structural and Multidisciplinary Optimization, vol. 60, no. 4, pp. 1475-1489, 2019.

[12] J. Ye and M. Lu, "Design optimization of domes against instability considering joint stiffness," Journal of Constructional Steel Research, vol. 169, Article ID 105757, 2020.

[13] L. X. Wang, W. F. Du, P. F. He, and M. Yang, "Topology optimization and 3D printing of three-branch joints in treelike structures," Journal of Structural Engineering, vol. 146, no. 01, Article ID 04019167, 2020.

[14] H. Seifi, A. Rezaee Javan, S. Xu, Y. Zhao, and Y. M. Xie, "Design optimization and additive manufacturing of nodes in gridshell structures," Engineering Structures, vol. 160, no. 04, pp. 161-170, 2018.

[15] J. Bai and W. Zuo, "Hollow structural design in topology optimization via moving morphable component method," Structural and Multidisciplinary Optimization, vol. 61, no. 1, pp. 187-205, 2020.

[16] W. J. Zuo, W. W. Li, T. Xu, S. Y. Xuan, and J. X. Na, "A complete development process of finite element software for body-in-white structure with semi-rigid beams in .NET framework," Advances in Engineering Software, vol. 45, no. 01, pp. 261-271, 2011.

[17] M. P. Bendsoe and N. Kikuchi, "Generating optimal topologies in structural design using a homogenization method," Computer Methods in Applied Mechanics and Engineering, vol. 71, no. 02 , pp. 197-224, 1988 .

[18] O. Sigmund and K. Maute, "Topology optimization approaches," Structural and Multidisciplinary Optimization, vol. 48, no. 6, pp. 1031-1055, 2013.

[19] Y. K. Sui and D. Q. Yang, "A new method for structural topological optimization based on the concept of independent continuous variables and smooth model," Acta Mechanica Sinica, vol. 14, no. 02, pp. 179-185, 1998.

[20] Y. M. Xie and G. P. Steven, "A simple evolutionary procedure for structural optimization,” Computers \& Structures, vol. 49, no. 5, pp. 885-896, 1993.

[21] Y. Tang, A. Kurtz, and Y. F. Zhao, "Bidirectional Evolutionary Structural Optimization (BESO) based design method for lattice structure to be fabricated by additive manufacturing," Computer-Aided Design, vol. 69, no. 06, pp. 91-101, 2015.

[22] M. Zhou and R. Gin, "Topological geometrical and generalized shape optimization," Computer Methods in Applied Mechanics and Engineering, vol. 89, no. 02, pp. 309-339, 1991.

[23] M. Stople and K. Svanberg, "An alternative interpolation scheme for minimum compliance topology optimization," Structural and Multidisciplinary Optimization, vol. 22, no. 02, pp. 116-124, 2001.

[24] Y. X. Li, Q. W. Yang, T. Chang, T. Qin, and F. H. Wu, "Multiload cases topological optimization by weighted sum method based on load case severity degree and ideality," Advances in Mechanical Engineering, vol. 12, no. 08, pp. 1-15, 2020.

[25] L. Rongrong, L. Yee, L. Hui, and H. Bo, “An adaptive compromise programming method for multiobjective path optimization," Journal of Geographical Systems, vol. 15, no. 02, pp. 211-228, 2013.

[26] G. A. kumar and G. Arumaikkannu, "Multiobjective topology optimization of additive manufactured alternator bracket," Materials Today: Proceedings, vol. 308, no. 10, pp. 1-5, 2020.

[27] I. Alessio, T. Menelaos, and M. Luis, "Analytic hierarchy process-fuzzy sorting: an analytic hierarchy process-based method for fuzzy classification in sorting problems," Journal of the Operational Research Society, vol. 71, no. 06, pp. 928-947, 2020.

[28] S. Nachiappan and R. Ramakrishnan, "A review of applications of Analytic Hierarchy Process in operations management," International Journal of Production Economics, vol. 138, no. 02, pp. 215-247, 2012.

[29] W. Zhong, R. Su, L. Gui, and Z. Fan, "Multiobjective topology and sizing optimization of bus body frame," Structural and Multidisciplinary Optimization, vol. 54, no. 3, pp. 701-714, 2016.

[30] S. J. Parkac, J. E. Leeb, H. B. Leeac et al., "3D printing of biobased polycarbonate and its potential applications in ecofriendly indoor manufacturing," Additive Manufacturing, vol. 31, no. 01, Article ID 100974, 2020.

[31] W. Jiang and Z. Fan, "Novel technologies for the lost foam casting process," Frontiers of Mechanical Engineering, vol. 13, no. 1, pp. 37-47, 2018.

[32] D. Thomas, "Costs, benefits, and adoption of additive manufacturing: a supply chain perspective," International Journal of Advanced Manufacturing Technology, vol. 85, no. 58, pp. 1857-1876, 2016. 